\title{
Misinterpretation of Negative Pulse Oximetry Screening as Absence of Critical Congenital Heart Disease
}

\author{
Krishna Dummula • Vishal Pandey
}

Received: 20 July 2012/Accepted: 7 August 2012/Published online: 23 August 2012

(C) Springer Science+Business Media, LLC 2012

We read the case report by Harden et al. [2] entitled FalseNegative Pulse Oximetry Screening for Critical Congenital Heart Disease: The Case for Parent Education with great interest. This case underscores the importance of interpreting screening test results with caution.

Since the endorsement of pulse oximetry screening (POS) for critical congenital heart disease (CCHD) by the American Academy of Pediatrics (AAP) [3], many hospitals across the country, including ours, have proactively implemented the screen. We applaud the AAP for the initiative and wish to highlight through this letter some of the practical concerns arising after implementation of these recommendations.

The AAP did not provide any standardized documentation guidelines for incorporating the screening results in the discharge summary to date. Nonstandard statements such as "CCHD screen-negative" in the nursery discharge summaries may give rise to a false sense of reassurance to the unfamiliar medical practitioner and the parents that the child has no possibility of critical congenital heart disease. The pulse oximetry screen holds a very high specificity for ruling out certain CCHDs, as demonstrated in the Swedish [1] and German [4] studies. Clearly, other conditions are associated with significant morbidity and mortality that need ongoing surveillance. Considering all these, we are concerned that there is room for overlooking serious congenital heart conditions if clinicians and parents are not adequately educated concerning the limitations of the screening protocol.

K. Dummula $\cdot$ V. Pandey $(\bowtie)$

School of Medicine, University of Kansas, 3901 Rainbow Boulevard, Mail Stop 2030, Kansas City, KS 66160, USA e-mail: vpandey@kumc.edu

K. Dummula · V. Pandey

The University of Kansas Hospital, Kansas City, KS, USA
We enthusiastically support the AAP's endorsement of pulse oximetry screening for early detection of CCHD but urge the Academy to mandate that nurseries document the cardiac conditions specifically ruled out by virtue of a negative screen on every discharge summary. We urge the Academy also to consider expanding the algorithm to include comprehensive guidelines for ongoing surveillance for congenital heart disease (CHD) during well-child visits of screen-negative children.

The AAP's recommendation calling for its members to work in concert with the local and regional chapters and respective policy makers in the development of the educational materials and training [3] is probably the best way to ensure that no child with CHD is missed.

Conflict of interest The authors declare that they have no conflict of interest.

\section{References}

1. de-Wahl Granelli A, Wennergren M, Sandberg K, Mellander M, Bejlum C, Inganas L, Eriksson M, Segerdahl N, Agren A, EkmanJoelsson BM, Sunnegardh J, Verdicchio M, Ostman-Smith I (2009) Impact of pulse oximetry screening on the detection of duct dependent congenital heart disease: a Swedish prospective screening study in 39,821 newborns. BMJ 338:a3037

2. Harden BW, Martin GR, Bradshaw EA (2012) False-negative pulse oximetry screening for critical congenital heart disease: the case for parent education. Pediatr Cardiol. doi:10.1007/s00246012-0414-5

3. Mahle WT, Martin GR, Beekman RH III, Morrow WR (2012) Endorsement of Health and Human Services recommendation for pulse oximetry screening for critical congenital heart disease. Pediatrics 129:190-192

4. Riede FT, Worner C, Dahnert I, Mockel A, Kostelka M, Schneider P (2010) Effectiveness of neonatal pulse oximetry screening for detection of critical congenital heart disease in daily clinical routine: results from a prospective multicenter study. Eur J Pediatr 169:975-981 